\title{
Shear isolation design combined with reinforcement blockage
}

\author{
Wang shuijiang
}

Sichuan College of Architectural Technology, Sichuan, 618000

Keywords: Sectional shape, reinforced blockade, shear isolation

Abstract. In the process of architectural design, sectional shape is used instead of original shape. Shear isolation design combined with reinforced blockade, make up for the lack of original wall bearing capacity, stiffness and ductility, energy, lack of hysteresis characteristics. In order to determine the effectiveness of shear isolation design under reinforced blockade. Building shear cracking damage experiment is performed. The results show that cross-sectional shape and reinforced shear wall buildings blockade isolation performance was significantly better than the original shape of the shear wall design, reinforced blockade is significantly enhanced, strength and ductility effect are dramatically improved.

\section{Introduction}

Nowadays, in the construction industry in our country, more attention was paid to the construction quality ${ }^{[1]}$. Systems of construction were relatively single, according to the situation of our country, most of them were using reinforced concrete structure ${ }^{[2,3]}$. According to the structure feature of the building, middle section of the vertical cross section were generally adopted, with the design of shear isolation, a relatively perfect in structure or relatively complete tube structure was formed ${ }^{[4-5]}$.

\section{Design of sectional shape and shear isolation under reinforced blockade}

Considering the problem of shear isolation design under reinforced blockade, design of sectional shape and shear isolation under reinforced blockade is proposed. Computation model of sectional shape and reinforced blockade is established. After using proportional dimension modeling, and considering building sectional shape of stress distribution, a new type of reinforcement distribution diversity model is adopted.

In architectural design, concrete used solid elements C4D6R, reinforcement used T3D2. Use isolated carrying capacity theory under reinforced blockade to combine steel and concrete, expressed as a compressed rigid composition, the adhesive in the process was ignored. The experiment model had two groups, namely $A$ and $B$, each group had four test pieces which used 1/5 proportional scale. Model code for group A is A1, A2, A3, and A4. Model code for group B is B1, B2, B3, and B4. Where group A is shear isolation model under original shape and ordinary steel, group B is sectional shape and shear isolation model under reinforced blockade. Consider the influence factors of construction mechanical loading method.

Modeling analysis equation is shown as following formula,

The first part is the axial pressure process analysis.

When $\mathrm{X} \leq 1$,

$$
\mathrm{Y}=\mathrm{T}_{1} \mathrm{X}+\left(4-3 \mathrm{~T}_{1}\right) \mathrm{X}^{2}+\left(\mathrm{T}_{1}-3\right) \mathrm{X}^{3}
$$

When $\mathrm{X}>1$,

$$
Y=\frac{X}{\left[T_{2}(X-2)^{3}+X^{2}\right]^{-1}}
$$

Where, T1, T2 is parameter values to determine rise or fall of axial stress by strain $\mathrm{Y}$ is axial compressive strength of reinforced concrete under blockade. 
Pressure stiffness of sectional shape is as following.

$$
\left.\mathrm{B}_{1=1 /\left(2 \mathrm{H}_{\alpha}\right.}+\frac{\lambda \mathrm{K}}{\mathrm{E}_{\alpha}}\right)
$$

Where $\mathrm{K}$ is height of shear isolation, $\mathrm{N}$ is inertia moment of the sectional shape.

$\lambda$ is the uneven distribution of stress factor. $\lambda=0.1, \mathrm{H}_{\alpha}, \mathrm{E}_{\alpha}$ are the modulus of elasticity and shear modulus of shear isolation. At the same time, isolation stress under reinforced blockade should be considered, when $\mathrm{A}_{\alpha} \leq \mathrm{A}_{0}$,

$$
\mathrm{B}_{2}=\mathrm{B} \kappa+n \mathrm{~A}_{\varepsilon} \mathrm{X}_{\varepsilon} \log 32 / \mathrm{I}_{\varepsilon}
$$

Where $\mathrm{B}_{\kappa}$ lateral stiffness under reinforced blockade, $\mathrm{A}_{\varepsilon}, \mathrm{I}_{\varepsilon}$ are the elastic modulus and strength under reinforced blockade. Before the cracking of specimen, stiffness would not change. $\mathrm{X}_{\varepsilon}$ is yield Strength of shear isolation under shear isolation.

The second part is following. Continually control the stress load building, when the building experiment enter the damage stage due to development of diagonal cracks, redistribution of internal forces will take place within the building. Thus reinforced block effect increases obviously, more horizontal load is taken, so that the sectional shape of the bearing capacity of the shear isolation decline has slowed, and deformation ability increases significantly. Therefore, isolated carrying capacity can be viewed as superposition of sectional shape and shear isolation under reinforced blockade, namely $\mathrm{B}_{\alpha}$.

$$
\left.\mathrm{B}_{\alpha=1 /(} \frac{\mathrm{K}^{2}}{2 \mathrm{H}_{\alpha}}+\frac{\lambda \mathrm{K}}{\mathrm{E}_{\alpha}}\right)+\mathrm{B}_{\kappa+n} \mathrm{~A}_{\varepsilon} \mathrm{X}_{\varepsilon} \log 32 / \mathrm{I}_{\varepsilon}
$$

It can be seen from the analysis of the above formulas, original shape and building isolation pressure of ordinary reinforcement fact is caused by ordinary reinforced concrete's lower performance shear plate diagonal crack entry point, which is brittleness corrupted and has lower isolation performance.

\section{Experiment analysis}

In order to verify the proposed shear isolation under reinforced blockade method, it is need to perform an experiment. In the experiment, the stiffness degradation graph of reinforced of concrete shear isolation model under original shape and ordinary steel and sectional shape and shear isolation under reinforced blockade is shown as below.

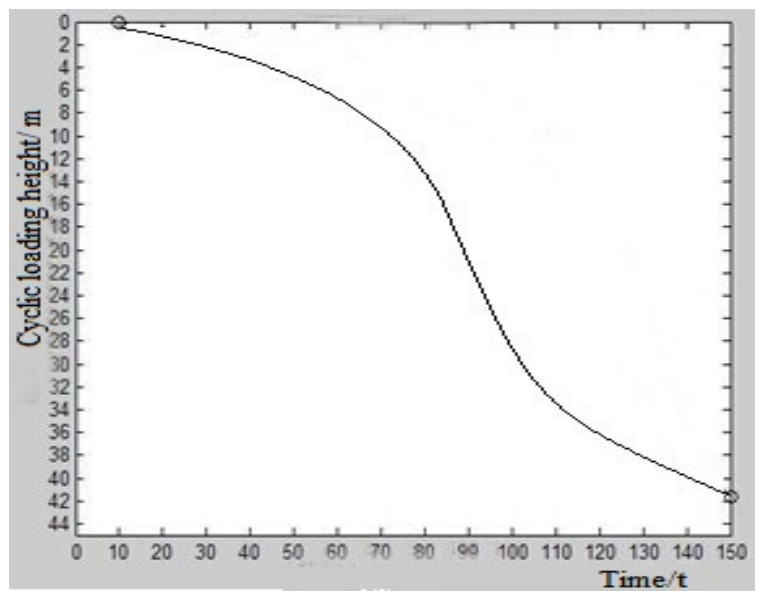

Figure 1. Stiffness degradation graph of reinforced concrete of original shear isolation structure 
Use the proposed shear isolation under reinforced blockade design, the corresponding stiffness degradation graph is as following.

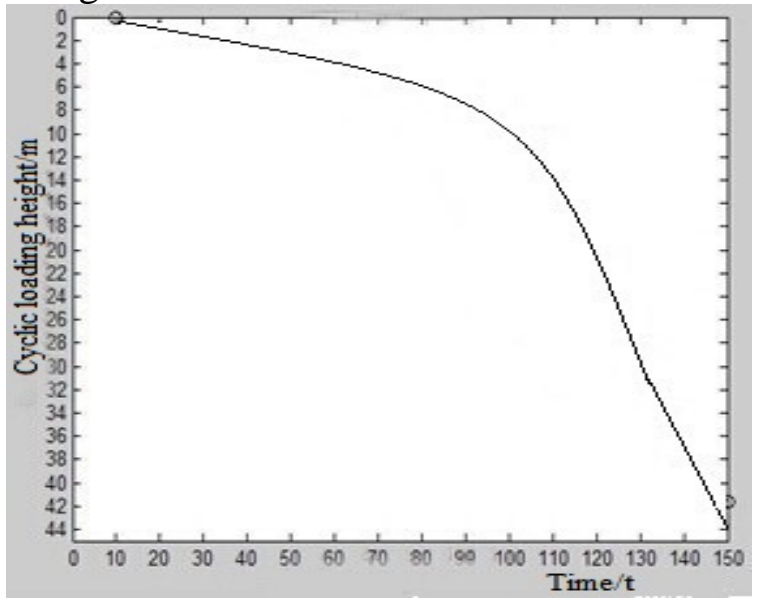

Figure 2. Stiffness degradation graph of reinforced concrete of proposed shear isolation structure

From the above stiffness degradation graphs, i.e. Figure 1 and Figure 2. For specimen A which is shear isolation model under original shape and ordinary steel and specimen B which is sectional shape and shear isolation model under reinforced blockade, the shear span ratios are different. The stiffness degradation rate is also almost the same trend. But if shear span ratios is relatively large, the stiffness of corresponding specimen with same angular displacement between layers increases.

\section{Conclusions}

From the experimental results, the shortage of shear isolation model under original shape and ordinary steel is more significant. In comparative experiment of modeling force loading method, analyze the stress of determined axial compressive stiffness ratio. Compressive strength has a significantly lower limit. The corresponding carrying capacity of shear isolation is limited. Especially when performing continuing controlled loading stress experiment, damage stage started from structural cracking to the maximum elastoplastic bearing stage, the shortage of shear isolation bearing load level exposed.

(1) The more carrying capacity of sectional shape and shear isolation under reinforced blockade is, the more significant ductility effect is. For carrying capacity from vertical load and the ones with strong pressure from all sides, the isolation effect is significant.

(2) Inside the ordinary construction structure, the carrying capacity of sectional shape is usually lower, which is often ignored. In the ordinary construction structure, the effect of carrying capacity of sectional shape is constantly increasing.

Based on the experimental and calculated results above, if deformation level of component is large, many adverse effects may occur. So it is required that, in the process of design, architecture stiffness control need to be paid more attention. Stiffness degradation rate of sectional shape and shear isolation under reinforced blockade is significant less, which means better performance.

\section{References}

[1] Cao Wanlin, Liu Chunyan, Zhang Jianwei, et al, Influence of inclination of the concealed bracing on the aseismic property of the R.C. shear wall [J]. World information on earthquake engineering, 2000,16(3):68-72.

[2] Industrial standard of the People's Republic of China, Technical specification for concrete structures of tall building [M],JGJ3-2002,J186-2002. 
[3] Yih-Lang Lin, Pe-i Yu Huang, Chih-Hong Hwang,et al.Performance and congestion-driven multilevel router[C].The 13th Workshop on Synthesis And System Integration of Mixed Information Technologies(SASIMI) 2006,2006-04.

[4] Lin S P,Chang Y W.A novel framework for multilevel routing cons-idering routability and performance[C] PPProc. of Int. Conf. Computer-Aided Design, November 2002:44-50.

[5] Zhang Yuming, Meng Shaoping, Qiu Ronggen, et al. Study on Prestressing Construction Questions of the Large Platform of Nanjing Olympic Sports Center [J].Construction technology,2005,34(7):13-15 